\title{
ON THE EVOLUTION OF AGE-STRUCTURED MARRIAGE FUNCTIONS: IT TAKES TWO TO TANGO*
}

November, 1995

BU-1310-M

\author{
Shu-Fang HSU SCHMITZ \\ Universität Bern \\ Inst. f. math. Statistik \\ Sidlerstrasse 5 \\ 3012 Bern, SWITZERLAND \\ Tel: $+41(31) 6318805$ \\ Fax: $+41(31) 6313870$ \\ e-mail: sfshsu@math-stat.unibe.ch \\ Carlos CASTILLO-CHAVEZ ${ }^{\dagger}$ \\ Biometrics Unit/Center for Applied Math. \\ 337 Warren Hall \\ Cornell University \\ Ithaca, NY 14853 \\ Tel: (607) $255-5488$ \\ Fax: (607) 255-4698 \\ e-mail: cc32@cornell.edu
}

* This paper is dedicated to the memory of Prof. Stavros Busenberg who passed away on April 3, 1993, and of Dr. Gail Rubin who died on February 7, 1995.

$\dagger$ To whom proofs and correspondence should be sent. 


\title{
ON THE EVOLUTION OF AGE-STRUCTURED MARRIAGE FUNCTIONS: IT TAKES TWO TO TANGO
}

\begin{abstract}
In prior work, we characterized two-sex marriage functions for socially structured populations as multiplicative perturbations of heterosexually random/proportionate mixing. These perturbations were expressed in terms of the preferences/affinities of males for females and vice versa. Male and female preferences/affinities are obviously not independent as they depend on the availability of male and female behavioral "genotypes." We show that knowledge of the preferences/affinities of one gender can characterize the preferences/affinities of both genders in socially-structured populations; in other words, it takes two to tango. This is the basic content of the $\mathrm{T}^{3}$ Theorem. In this chapter, we revise our results for socially structured populations and extend them to situations where the population is characterized by continuous variables such as age. It is shown that different sets of preferences/affinities, that is, distinct behavioral "genotypes", may give rise to identical mixing/mating probabilities, the determinants of the behavioral "phenotypes." Hence, different sets of individual decisions can lead to identical social dynamics - a fact well established in genetics. The importance of the incorporation of mating systems at the population level is a neglected but central area in evolutionary biology.
\end{abstract}




\section{Introduction}

Marriage functions are solutions to the two-sex mixing/pairing problem. Despite their importance in areas such as population genetics (mating functions), demography (population projection), cultural anthropology (preservation and dissemination of cultural traits), and evolutionary biology (life history), their application has been quite limited. Most researchers have addressed theoretical issues in these areas through the use of single-sex models or highly simplified two-sex models. A basic premise being ignored is that "it takes two to tango." The difficulties involved are quite evident from the pioneering work of Kendall (1949), Keyfitz (1949), Fredrickson (1971), McFarland (1972), Parlett (1972), Pollard (1973), and Caswell and Weeks (1986).

We have developed an axiomatic framework to conduct a systematic study of marriage functions (Busenberg and Castillo-Chavez 1989, 1991; Castillo-Chavez and Busenberg 1991; Hsu Schmitz 1994; Hsu Schmitz et al. 1994, Blythe et al. 1991. Our work has been applied in areas as diverse as cultural anthropology (Lubkin and Castillo-Chavez 1995), demography (Castillo-Chavez, Fridman and Luo 1995), epidemiology and food web dynamics (Castillo-Chavez, Velasco-Hernandez and Fridman 1994), and parameter estimation (Castillo-Chavez et al. 1992, Hsu Schmitz and Castillo-Chavez 1994, 1995). We provide a summary of our characterization of marriage functions for populations defined through fixed characteristics such as race, language, biological species, religion, level of education, and socioeconomic level. We then provide a detailed characterization of age-structured marriage/mixing functions. The discrete framework described in this chapter can be incorporated into finite dimensional deterministic or stochastic models while the continuous framework is easily incorporated into age structured models.

In earlier work, we (Castillo-Chavez and Busenberg 1991) characterized two-sex marriage functions as multiplicative perturbations of the Ross solution, that is, heterosexually random or proportionate mixing. These perturbations were defined in terms of the preferences/affinities of males for females and vice versa. Male and female preferences depend on the availability and frequency of male and female behavioral "genotypes". Our key result states that knowledge of the preferences/affinities of one gender can characterize the preferences/affinities of both genders; in other 
words, it takes two to tango (the $\mathrm{T}^{3}$ Theorem). In Hsu Schmitz (1994) and Hsu Schmitz et al. (1994) we also showed that different sets of preferences/affinities, that is, distinct behavioral "genotypes", may give rise to identical mixing/mating probabilities. Hence, different sets of individual decisions can lead to identical social dynamics.

A useful interpretation of our work is to think of preferences/affinities as a method for parameterizing a family of conditional probability distributions. Then our main result establishes that the parameterization of the male distribution completely determines (parameterizes) its associated female distribution and vice versa. In fact, the $\mathrm{T}^{3}$ Theorem makes it possible to generate new parametric families of distributions (marriage functions) systematically. One-, two-, or n-parameter mixing distributions can be easily constructed to model a pre-specified set affinities associated with a two-sex mating system. For example, the two-parameter family of functions given by $\phi\left(a, a^{\prime}\right)=\nu$ $\exp \left[-\kappa\left(a-a^{\prime}\right)^{2}\right]$, which would model like-with-like or preferred affinities between males of age $a$ and females of age $a^{\prime}$, can be easily incorporated into dynamic two-sex models via our two-parameter agestructured marriage/mixing function with like-with-like preferences given by $\phi\left(a, a^{\prime}\right)$. Structured population models that explicitly incorporate mating functions of this type at the population level have never been studied. Hence the role of selection in mating systems at the population level has not been properly explored.

This chapter is organized as follows: Section 2 introduces a two-sex mixing framework for a population defined in terms of fixed characteristics based on our earlier work (Castillo-Chavez and Busenberg 1991, Rubin et al. 1992); Section 3 introduces flexible parametric families of mixing solutions that make connections to data possible; Section 4 discusses the relationship between males and females preferences/affinities through the $\mathrm{T}^{3}$ theorem; Section 5 establishes analogous results in a continuous framework; Section 6 summarizes our results.

\section{Discrete Two-sex Mixing Framework}

Consider a population with $L$ types of males and $N$ types of females. Let $T_{i}^{m}(t)$ denote the number of males of type $i(i=1, \ldots, L)$ at time $t$, and $T_{j}^{f}(t)$ denote the number of females of type $j$ 
$(j=1, \ldots, N)$ at time $t$. Let $C_{i}(i=1, \ldots, L)$ and $B_{j}(j=1, \ldots, N)$ denote the per capita pairing rates for males of type $i$ and for females of type $j$, respectively. These rates must in general be assumed to be functions of the state variables $\mathbf{T}^{m}=\left(T_{1}^{m}, \ldots, T_{L}^{m}\right)^{\mathrm{T}}$ and $\mathbf{T}^{f}=\left(T_{1}^{f}, \ldots, T_{N}^{f}\right)^{\mathrm{T}}$. This is an important assumption as the absence of either males or females makes mating impossible, that is, if one sex is not present these rates must be zero.

We characterize two-sex marriage/mixing functions using two matrices: $\mathbf{P}(\cdot, t)=\left\{p_{i j}(\cdot, t)\right\}$ and $\mathrm{Q}(\cdot, t)=\left\{q_{j i}(\cdot, t)\right\}$. Here $p_{i j}(\cdot, t)$ denotes the conditional probability that a male of type $i$ pairs with a female of type $j$ given that he has formed a heterosexual partnership at time $t$ while $q_{j i}(\cdot, t)$ denotes the conditional probability that a female of type $j$ pairs with a male of type $i$ given that she has formed a heterosexual partnership at time $t$. These matrices are functions of average weighted pairing rates of the various groups, that is, they are functions of the "abundance" of partners and their affinities for particular types. The pair $(\mathbf{P}(\cdot, t), \mathbf{Q}(\cdot, t))$ is called a marriage or mixing/pair-formation matrix if and only if it satisfies the following properties at all times (more general properties that include those below can be found in Castillo-Chavez, Huang and Li 1994) :

$$
\begin{aligned}
& p_{i j}(\cdot, t) \geq 0 \text { and } q_{j i}(\cdot, t) \geq 0 \text { for } i=1, \ldots, L, j=1, \ldots, N . \\
& \sum_{j=1}^{N} p_{i j}(\cdot, t)=1 \text { for } i=1, \ldots, L ; \sum_{i=1}^{L} q_{j i}(\cdot, t)=1 \text { for } j=1, \ldots, N . \\
& C_{i}\left(\mathbf{T}^{m}, \mathbf{T}^{f}\right) T_{i}^{m}(t) p_{i j}(\cdot, t)=B_{j}\left(\mathbf{T}^{m}, \mathbf{T}^{f}\right) T_{j}^{f}(t) q_{j i}(\cdot, t) \text { for } i=1, \ldots, L, j=1, \ldots, N .
\end{aligned}
$$

Note that (A2) and (A3) imply

$$
\sum_{i=1}^{L} C_{i}\left(\mathbf{T}^{m}, \mathbf{T}^{f}\right) T_{i}^{m}(t)=\sum_{j=1}^{N} B_{j}\left(\mathbf{T}^{m}, \mathbf{T}^{f}\right) T_{j}^{f}(t)
$$

which in fact provides a necessary and sufficient condition to guarantee the existence of a solution. The only separable solution (Castillo-Chavez and Busenberg 1991), to axioms (A1)-(A3) is the Ross solution: $p_{i j}=\bar{p}_{j}$ and $q_{j i}=\bar{q}_{i}$, where

$$
\bar{p}_{j}=\frac{B_{j} T_{j}^{f}}{\sum_{j=1}^{N} B_{j} T_{j}^{f}} \quad \text { and } \quad \bar{q}_{i}=\frac{C_{i} T_{i}^{m}}{\sum_{i=1}^{L} C_{i} T_{i}^{m}} .
$$


Castillo-Chavez and Busenberg (1991) characterized all solutions to axioms (A1)-(A3) as multiplicative perturbations of the Ross solution. These perturbations were defined in terms of two matrices: $\boldsymbol{\Phi}^{m}=\left\{\phi_{i j}^{m}\right\}$ and $\boldsymbol{\Phi}^{f}=\left\{\phi_{j i}^{f}\right\}$. The matrices $\boldsymbol{\Phi}^{m}$ and $\Phi^{f}$ are a measure of the mating preferences and/or affinities of individuals of each gender for the the opposite gender. These preferences may change with time directly, through changes in the frequency of mixing types, or through changes in the abundance of each type. We call them the male and female preference matrices, respectively. To explicitly state our prior characterization theorem of two-sex marriage functions we need some definitions:

$$
\begin{array}{rlrl}
\ell_{i}^{m} & \equiv \sum_{j=1}^{N} \bar{p}_{j} \phi_{i j}^{m}, & R_{i}^{m} \equiv 1-\ell_{i}^{m}, & V^{m} \equiv \sum_{i=1}^{L} \bar{q}_{i} R_{i}^{m}, \\
\ell_{j}^{f} \equiv \sum_{i=1}^{L} \bar{q}_{i} \phi_{j i}^{f}, & R_{j}^{f} \equiv 1-\ell_{j}^{f}, & V^{f} \equiv \sum_{j=1}^{N} \bar{p}_{j} R_{j}^{f} .
\end{array}
$$

Theorem 1. (Castillo-Chavez and Busenberg 1991)

For each marriage function $(\mathbf{P}, \mathbf{Q})$ matrices $\mathbf{\Phi}^{m}$ and $\Phi^{f}$ can be found so that

$$
p_{i j} \equiv \bar{p}_{j}\left[\frac{R_{j}^{f} R_{i}^{m}}{V^{f}}+\phi_{i j}^{m}\right] \quad \text { and } \quad q_{j i} \equiv \bar{q}_{i}\left[\frac{R_{i}^{m} R_{j}^{f}}{V^{m}}+\phi_{j i}^{f}\right]
$$

where $0 \leq R_{i}^{m} \leq 1 \quad(i=1, \ldots, L), \quad 0 \leq R_{j}^{f} \leq 1 \quad(j=1, \ldots, N), \quad \sum_{i=1}^{L} \ell_{i}^{m} \bar{q}_{i}<1$ and $\sum_{j=1}^{N} \ell_{j}^{f} \bar{p}_{j}<1$ if and only if

$$
\phi_{i j}^{m}=\phi_{j i}^{f}+R_{i}^{m} R_{j}^{f}\left[\frac{1}{V^{m}}-\frac{1}{V^{f}}\right]
$$

Condition (5) shows the implicit frequency and time dependent relationship forced by (A3) between the elements of $\boldsymbol{\Phi}^{m}$ and $\boldsymbol{\Phi}^{f}$. Letting

$$
\overrightarrow{\overline{\mathbf{p}}}=\left(\begin{array}{c}
\bar{p}_{1} \\
\vdots \\
\bar{p}_{N}
\end{array}\right) \quad \text { and } \quad \overrightarrow{\overline{\mathbf{q}}}=\left(\begin{array}{c}
\bar{q}_{1} \\
\vdots \\
\bar{q}_{L}
\end{array}\right)
$$

and using matrix notation, we can combine the constraints imposed by (5) into an implicit non-linear relationship of the form 


$$
\Phi^{m}=\Psi\left(\overrightarrow{\overline{\mathbf{p}}}, \overrightarrow{\overline{\mathbf{q}}}, \Phi^{f} \cdot \Phi^{m}\right)
$$

where the elements of $\psi$ are defined component-wise by (5). The non-linear expression (6) succinctly summarizes the constraints imposed by (A3) on the mixing sub-populations and their defining parameters. The representation theorem was built without explicitly determining the class of matrices $\Phi^{m}$ and $\Phi^{f}$ that may result naturally from the mixing constraints imposed by heterosexually mixing populations. The natural reduction on the number of degrees of freedom available to a heterosexually mixing population is not completely obvious and, in fact, is the content of the $\mathrm{T}^{3}$ Theorem.

The roles of the affinity matrices are not entirely clear from the above result. A naive interpretation when it is assumed that both are matrices of constants may lead to the wrong, and deceptive, interpretation that all we have managed to do is to parametrize a model (see Altman and Morris 1994). A clearer understanding of this model can be seen directly from Blythe et al. (1995 paper, which was used-including some of its results - by Altman and Morris as the basis for their paper and never cited!). A preliminary result which gives an insight into the role of $\boldsymbol{\Phi}^{m}$ and $\boldsymbol{\Phi}^{f}$ is the following:

Theorem 2. (Castillo-Chavez and Busenberg 1991) If either $\phi_{i j}^{m}=\alpha, \quad 0 \leq \alpha<1 \quad \forall i, j, \quad$ or $\phi_{j i}^{f}=\beta, \quad 0 \leq \beta<1 \quad \forall j, i$, where $\alpha$ and $\beta$ are constants, then $p_{i j}=\bar{p}_{j}$ and $q_{j i}=\bar{q}_{i}$. That is, equation (4) reduces to the unique separable Ross solution in (2).

Theorem 2 says that in a mating system whose either males or females have identical affinitites then random/proportionate two-sex mixing is the only possibility. Other models are pursued in Section 3.

\section{Parameterization of Preference Matrices}

Equation (4) encapsulates all possible mixing patterns in terms of two preference matrices. It may be argued, to some degree correctly, that this representation transfers the difficulties from one set 
of matrices, $(\mathbf{P}, \mathbf{Q})$, to another, $\left(\Phi^{m}, \Phi^{f}\right)$. However, as we show later on, the use of preference matrices $\left(\Phi^{m}, \Phi^{f}\right)$ increases our understanding of the marriage/social structure of a population. Specific preference matrices facilitate the modeling of non-trivial parametric mixing patterns (again see Blythe et al. 1995). Indeed, it is possible to construct easily a large class of marriage functions that go well beyond those found in the literature, a class of functions that allows for the clear modeling of, for example, asymmetric age-structured interactions. Asymmetric age-structured interactions (younger females dating older males) while common in human populations have never been incorporated in ordinary age-structured pairing models because of the lack of an appropriate model. From the results of this paper, it will be obvious how to construct mating systems that incorporate realistic affinities with few parameters.

Most of the prior theoretical work on mating systems was based on random, or specific types of assortative mating with some minor variations. This is particularly clear from the literature in population genetics (Crow and Kimura 1970). Modelers interested in mating systems at the population level began to move away from random mating through the use of special mixing matrices including like-with-like, preferred mixing, or biased mixing (see Sattenspiel and Castillo-Chavez 1989). Other forms of mixing such as asymmetric heterosexual mixing, where females prefer to mix with older males and males prefer to mix with younger females have been avoided because the lack of a clear modeling framework. The theoretical consequences associated with this type of limitations are evident from the literature on marriage functions, demography, and mathematical demography. Furthermore, a glance at the literature on mathematical ecology and epidemiology shows that efforts to understand disease dynamics and demographic effects in gender-specific populations have been conducted using mostly unrealistic mating structures. We must therefore ask the obvious question: to what extent is our current theoretical understanding of mating systems or of population dynamics solely dependent on our use of specialized forms of mixing? The use of our preference matrices $\left(\Phi^{m}, \Phi^{f}\right)$ makes the construction of flexible and parameter-poor mating systems possible. Future applications of these models will measure the success/utility of this approach. 
A simple result that allows for the explicit construction of flexible mixing/mating parameter-poor structures is the following:

Theorem 3. $V^{f}=V^{m}$ if and only if $\Phi^{m}=\left(\Phi^{f}\right)^{\mathrm{T}}$, where $\mathrm{T}$ denotes transposition.

Theorem 3 says that the only solutions to axioms (A1)-(A3) with frequency independent $\Phi^{m}$ and $\Phi^{f}$ are those with $\Phi^{m}=\left(\Phi^{f}\right)^{\mathrm{T}}$. This simple case corresponds to the situation where males and females have matching and fixed preferences (preferences that do not change with the dynamics of $T_{i}^{m}(t)$ and $\left.T_{j}^{f}(t)\right)$. The class of solutions with $\Phi^{m}=\left(\Phi^{f}\right)^{\mathrm{T}}$ is restrictive, yet they help define parametrically the mixing/mating structures available in the literature with a limited number of parameters, as many of the entries can be (and should be if the model is to be of practical use) filled with zeros. The use of preference matrices $\Phi^{m}$ and $\Phi^{f}$ with constant entries provides a class of parametric mixing distributions that are rich, flexible, and connectable to data. We illustrate the use of these matrices with data derived by Rubin et al. (1992), Hsu Schmitz (1994), and Hsu Schmitz and Castillo-Chavez (1994).

Figure 1 illustrates a mixing matrix exhibiting like-with-like mixing (individuals prefer to mix with those of the same class or age) coupled with an additional trend, where females tend to pair with older males and males tend to pair with younger females. Thus, the use of constant preference matrices that satisfy the relationship $\Phi^{m}=\left(\Phi^{f}\right)^{\mathrm{T}}$ provides a reasonable mixing parametric model that captures this mating structure with only a "couple" of parameters - regardless of the complexity of the social structure of the population defined as the number of classes or categories. Here the number of classes is fixed. When a population is classified by age then we will have dynamic categories.

$$
\text { Figure } 1 \text { about here }
$$

Figure 2 about here

Figure 2 shows the corresponding random mixing pattern associated with the same parameters. To put emphasis on the flexibility of our approach we observe that we could model fit the data of Figure 1 with a one-parameter class of mixing matrices. In fact, Hsu Schmitz (1994) uses the following $\Phi^{f}:$ 


$$
\boldsymbol{\Phi}^{f}=\left[\begin{array}{lllll}
1 & d & d & d & d \\
0 & 1 & d & d & d \\
0 & 0 & 1 & d & d \\
0 & 0 & 0 & 1 & d \\
0 & 0 & 0 & 0 & 1
\end{array}\right]
$$

If the preference matrix $\Phi^{m}=\left(\Phi^{f}\right)^{\mathrm{T}}$ is fixed, that is, if all their elements are constant, then relationship (5) is always satisfied. Therefore, once we have computed an estimate $\hat{d}$ of $d$ from data, we can predict $p_{i j}(\hat{d},$.$) and q_{j i}(\hat{d},$.$) for all future times using (4) or (21) and a model, deterministic$ or stochastic, for the dynamics of the populations $T_{i}^{m}(t)$ and $T_{j}^{f}(t)$. Mixing matrices parametrized in the above fashion are flexible enough to capture the qualitative features observed in the data with a single parameter, $d$. Hence the assumption that $\Phi^{m}=\left(\Phi^{f}\right)^{\mathrm{T}}$ makes it possible to construct mixing/mating functions modeled by few parameters. Further applications/extensions are possible. We (Castillo-Chavez, Fridman and Luo 1995) have constructed stochastic and deterministic demographic and epidemiological models that incorporate the contact structure just described. We now proceed to discuss the key modeling result of this chapter.

\section{The Two Body Problem in a Discrete Framework}

In Section 3, we found that the preferences of males for females and vice versa satisfy in general a complex relationship, namely,

$$
\Phi^{m}=\Psi\left(\overrightarrow{\overline{\mathbf{p}}}, \overrightarrow{\overline{\mathbf{q}}}, \Phi^{f}, \Phi^{m}\right) .
$$

Common sense dictates that if one set of preferences (e.g., $\left.\Phi^{f}\right)$ is known then so must be the other (e.g., $\left.\boldsymbol{\Phi}^{m}\right)$. Consequently, it should be possible to solve (5) in terms of a single affinity matrix. Therefore, we expect that a functional $\Psi$ can be found so that

$$
\Phi^{m}=\Psi\left(\overrightarrow{\overline{\mathbf{p}}}, \overrightarrow{\overline{\mathbf{q}}}, \Phi^{f}\right)
$$

This result is the main content of our $\mathrm{T}^{3}$ Theorem. 
To find a solution of $\Phi^{m}$ in terms of $\bar{p}_{j}, \bar{q}_{i}$ and $\phi_{j i}^{f}$, we multiply $\bar{p}_{j}$ on both sides of equation (5) and sum over $j$. The resulting relationships are:

$$
\begin{aligned}
& \sum_{j=1}^{N} \bar{p}_{j} \phi_{i j}^{m}=\sum_{j=1}^{N} \bar{p}_{j} \phi_{j i}^{f}+\sum_{j=1}^{N} \bar{p}_{j} R_{i}^{m} R_{j}^{f}\left[\frac{1}{V^{m}}-\frac{1}{V^{f}}\right] \\
\Leftrightarrow & 1-R_{i}^{m}=\sum_{j=1}^{N} \bar{p}_{j} \phi_{j i}^{f}+R_{i}^{m} V^{f}\left[\frac{1}{V^{m}}-\frac{1}{V^{f}}\right] \\
\Leftrightarrow & 1-\sum_{j=1}^{N} \bar{p}_{j} \phi_{j i}^{f}=R_{i}^{m}\left[\frac{V^{f}}{V^{m}}\right] .
\end{aligned}
$$

Let

$$
U_{i}^{f} \equiv \sum_{j=1}^{N} \bar{p}_{j} \phi_{j i}^{f}
$$

then from equation (7) we obtain

$$
\frac{1-U_{i}^{f}}{V^{f}}=\frac{R_{i}^{m}}{V^{m}}
$$

which reveals the fact that male preferences can be obtained from female preference if we can solve (9). If we define

$$
\beta_{i}^{f} \equiv \frac{1-U_{i}^{f}}{V^{f}}
$$

then the system that must be solved becomes

$$
R_{i}^{m}-\beta_{i}^{f} V^{m}=0
$$

or

$$
R_{i}^{m}-\beta_{i}^{f} \sum_{k=1}^{L} \bar{q}_{k} R_{k}^{m}=0
$$

It can also be formulated in matrix notation as follows:

$$
\left(\mathrm{I}-\overrightarrow{\boldsymbol{\beta}}^{f} \overrightarrow{\mathbf{q}}^{\mathrm{T}}\right) \overrightarrow{\mathbf{R}}^{m}=\overrightarrow{\mathbf{0}}
$$

where

$$
\overrightarrow{\boldsymbol{\beta}}^{f}=\left(\begin{array}{c}
\beta_{1}^{f} \\
\vdots \\
\beta_{L}^{f}
\end{array}\right) \quad \text { and } \quad \overrightarrow{\mathbf{R}}^{m}=\left(\begin{array}{c}
R_{1}^{m} \\
\vdots \\
R_{L}^{m}
\end{array}\right)
$$

If we let $\mathbf{B}=\mathbf{I}-\overrightarrow{\boldsymbol{\beta}}^{f} \overrightarrow{\overrightarrow{\mathbf{q}}}^{\mathrm{T}}$, then we observe that $\mathbf{B}$, an $L \times L$ matrix, is a rank one perturbation of the identity. Furthermore, a simple computation shows that

$$
\operatorname{det} \mathbf{B}=1-\sum_{i=1}^{L} \bar{q}_{i} \beta_{i}^{f}=0,
$$


and hence all solutions are given by

$$
\overrightarrow{\mathbf{R}}^{m}=\gamma \overrightarrow{\boldsymbol{\beta}}^{f}
$$

where $\gamma$ is an arbitrary "constant" for each time $t$. In other words, the null space of $\mathbf{I}-\overrightarrow{\boldsymbol{\beta}}^{f} \overrightarrow{\overrightarrow{\mathbf{q}}}^{\mathrm{T}}$ is equal to the $\operatorname{span}\left\{\overrightarrow{\boldsymbol{\beta}}^{f}\right\}$. Substituting solution (12) into (5) gives the relationship

$$
\phi_{i j}^{m}=\phi_{j i}^{f}+\gamma \beta_{i}^{f} R_{j}^{f}\left[\frac{1}{\gamma}-\frac{1}{V^{f}}\right]=\phi_{j i}^{f}+\beta_{i}^{f} R_{j}^{f}\left[1-\frac{\gamma}{V^{f}}\right]
$$

The condition $\sum_{j=1}^{N} \ell_{j}^{f} \bar{p}_{j}<1$ in Theorem 1 implies $\sum_{i=1}^{L} \bar{q}_{i} U_{i}^{f}<1$ because

$$
\sum_{j=1}^{N} \ell_{j}^{f} \bar{p}_{j}=\sum_{j=1}^{N} \sum_{i=1}^{L} \bar{q}_{i} \phi_{j i}^{f} \bar{p}_{j}=\sum_{i=1}^{L} \bar{q}_{i} U_{i}^{f}
$$

If we further constrain $\phi_{j i}^{f}$ by requiring that $U_{i}^{f}=\sum_{j=1}^{N} \bar{p}_{j} \phi_{j i}^{f} \leq 1$ then $\beta_{i}^{f} \geq 0$ for all $i$ (by its own definition) as negative values of $\beta_{i}^{f}$ would imply that $U_{i}^{f}>1$. Finally, in order to have $0 \leq R_{i}^{m} \leq 1$, we need to choose $\gamma$ satisfying

$$
0 \leq \gamma \leq \frac{1}{\max _{i} \beta_{i}^{f}}
$$

Not all $\beta_{i}^{f}$ can be zero, or not all $U_{i}^{f}$ can be one, otherwise $V^{f}=0$. The parameter $\gamma$ gives an extra degree of freedom in the choice of $R_{i}^{m}$ and $\phi_{i j}^{m}$. To simplify the expression, we reparameterize or rescale

the free parameter $\gamma$ and let

$$
\Gamma \equiv 1-\frac{\gamma}{V^{f}},
$$

so

$$
\gamma=V^{f}(1-\Gamma)
$$

Hence, equation (13) becomes

$$
\begin{aligned}
\phi_{i j}^{m} & =\phi_{j i}^{f}+\beta_{i}^{f} R_{j}^{f}\left[1-\frac{V^{f}(1-\Gamma)}{V^{f}}\right]=\phi_{j i}^{f}+\Gamma \beta_{i}^{f} R_{j}^{f} \\
& =\phi_{j i}^{f}+\Gamma \frac{\left(1-U_{i}^{f}\right) R_{j}^{f}}{V^{f}} .
\end{aligned}
$$


Plugging equation (15) into inequality (14) results in

$$
\begin{array}{ll} 
& 0 \leq V^{f}(1-\Gamma) \leq \frac{1}{\max _{i} \beta_{i}^{f}} \\
\Leftrightarrow & 1-\frac{1}{V^{f} \max _{i} \beta_{i}^{f}} \leq \Gamma \leq 1, \\
\Leftrightarrow & \frac{-\min _{i} U_{i}^{f}}{1-\min _{i} U_{i}^{f}} \leq \Gamma \leq 1 .
\end{array}
$$

These computations allow us to state our main result, the $\mathrm{T}^{3}$ Theorem:

Theorem 4. (discrete $\mathbf{T}^{3}$ Theorem)

The preference matrices at all times obey the explicit relation

$$
\phi_{i j}^{m}=\phi_{j i}^{f}+\Gamma \frac{\left(1-U_{i}^{f}\right) R_{j}^{f}}{V^{f}},
$$

where $\Gamma$ is an implicitly time-dependent arbitrary "constant" satisfying

$$
\frac{-\min _{i} U_{i}^{f}}{1-\min _{i} U_{i}^{f}} \leq \Gamma \leq 1 \text { and } U_{i}^{f} \leq 1
$$

and conversely

$$
\phi_{j i}^{f}=\phi_{i j}^{m}+\Delta \frac{\left(1-U_{j}^{m}\right) R_{i}^{m}}{V^{m}}
$$

where

$$
U_{j}^{m}=\sum_{i=1}^{L} \bar{q}_{i} \phi_{i j}^{m} \leq 1
$$

and $\Delta$ is an implicitly time-dependent arbitrary "constant" satisfying

$$
\frac{-\min _{j} U_{j}^{m}}{1-\min _{j} U_{j}^{m}} \leq \Delta \leq 1 .
$$

The function $\Psi$ given by $(*)$ is thus defined explicitly by equation (17). Yes, the above result shows that the original model was overparameterized (assumes constant affinities). However, by explicitly establishing the relationships between female and male affinities, we are able to construct parametric 
families of mating functions that capture a rich variety of situations never found in the literature, and, need be, with few parameters.

If $\phi_{j i}^{f}=\alpha$ (constant, and $0 \leq \alpha<1$ ) for all $i, j$, then $0<R_{j}^{f}=1-\alpha \leq 1$ for all $j$, $0<V^{f}=1-\alpha \leq 1$ by (3), and $0 \leq U_{i}^{f}=\alpha<1$ for all $i$ by (8). Thus, $\beta_{i}^{f}=\frac{1-\alpha}{1-\alpha}=1$ by (10) and $0 \leq R_{i}^{m}=\gamma \leq 1$ by (12) for all $i$. Hence, from (13)

$$
\phi_{i j}^{m}=\alpha+(1-\alpha)\left[1-\frac{\gamma}{1-\alpha}\right]=1-\gamma=\mathrm{constant}
$$

for all $i, j$, and $0 \leq 1-\gamma<1$. Hence, lack of selectivity (preference) in one sex implies lack of selectivity (preference) in the other (Theorem 2). In this case, $p_{i j}=\bar{p}_{j}$ and $q_{j i}=\bar{q}_{i}$, that is, the population mixes at random (Ross solutions, simple versions used by Ross in his malaria work, see Blythe et al. 1992). If $\Gamma=0$ or $\Delta=0$ for all times, then $\Phi^{m}=\left(\Phi^{f}\right)^{\mathrm{T}}$ and we recover the frequency independent mixing matrices of Theorem 3.

Using the mixing solution given by (12) and (13), the general mixing matrix in (4) can be rewritten as follows:

$$
\begin{aligned}
& p_{i j}=\bar{p}_{j}\left[\beta_{i}^{f} R_{j}^{f}+\phi_{j i}^{f}\right], \\
& q_{j i}=\bar{q}_{i}\left[\beta_{i}^{f} R_{j}^{f}+\phi_{j i}^{f}\right]
\end{aligned}
$$

If we visualize the preference matrices $\Phi^{m}$ and $\Phi^{f}$ as behavioral "genotypes" then (21) which expresses a mating system as a function of behavioral "genotypes" is independent of $\gamma$. Therefore, behavioral " $\gamma$ genotypes" can give rise to identical behavioral "phenotypes" or equivalently the same set of mixing probabilities. In other words, the matching relations set by axioms (A1)-(A3) show that (21) gives a consistent reparametrization of the initial model. The fact that the same set of parameters give rise to identical mating systems can be interpreted just as the result of an over-parameterized model (an issue that we have addressed above). However, the possibility that the different sets of biological parameters give rise to the same observed behaviors (mating systems) is common in biology and, in some sense, the essence behind the definition of phenotype (an equivalence class that expresses the observable characteristics of distinct genotypes). Section 5 extends these results to age-structured populations. 


\section{Two-sex Mixing Framework in Age-structured Populations}

To describe the two-sex mixing framework in an age-structured population, we use the following notation:

$$
\begin{aligned}
M(a, t)= & \text { number of males with age in }[a, a+\Delta a] \text { at time } t, \\
F\left(a^{\prime}, t\right)= & \text { number of females with age in }\left[a^{\prime}, a^{\prime}+\Delta a\right] \text { at time } t, \\
C(a, \cdot)= & \text { per capita pairing rate for males of age } a, \\
B\left(a^{\prime}, \cdot\right)= & \text { per capita pairing rate for females of age } a^{\prime}, \\
p\left(a, a^{\prime}, t, \cdot\right)= & \text { probability that a male of age } a \text { pairs with a female of age } a^{\prime} \\
& \text { given that he pairs at time } t \\
q\left(a^{\prime}, a, t, \cdot\right)= & \text { probability that a female of age } a^{\prime} \text { pairs with a male of age } a \\
& \text { given that she pairs at time } t
\end{aligned}
$$

where $a, a^{\prime}, t \in[0, \infty)$, and $C(a, \cdot)$ and $B\left(a^{\prime}, \cdot\right)$ are assumed to be functions of $T^{m}(t) \equiv \int_{0}^{\infty} M(a, t) \mathrm{d} a$ and $T^{f}(t) \equiv \int_{0}^{\infty} F\left(a^{\prime}, t\right) \mathrm{d} a^{\prime}$. Castillo-Chavez, Busenberg, and Gerow (1991) state that two-sex mixing functions $(p, q)$ must satisfy the following properties at all times:

(B1) $p\left(a, a^{\prime}, t, \cdot\right) \geq 0$ and $q\left(a^{\prime}, a, t, \cdot\right) \geq 0$ for all $a$ and $a^{\prime}$,

(B2) $\int_{0}^{\infty} p\left(a, a^{\prime}, t, \cdot\right) \mathrm{d} a^{\prime}=1$ for all $a$, and $\int_{0}^{\infty} q\left(a^{\prime}, a, t, \cdot\right) \mathrm{d} a=1$ for all $a^{\prime}$,

(B3) $C(a, \cdot) M(a, t) p\left(a, a^{\prime}, t, \cdot\right)=B\left(a^{\prime}, \cdot\right) F\left(a^{\prime}, t\right) q\left(a^{\prime}, a, t, \cdot\right)$ for all $a$ and $a^{\prime}$.

These properties should be stated as holding almost everywhere. However, these technicalities are avoided in this chapter. Note that (B2) and (B3) imply that

$$
\int_{0}^{\infty} C(a, \cdot) M(a, t) \mathrm{d} a=\int_{0}^{\infty} B\left(a^{\prime}, \cdot\right) F\left(a^{\prime}, t\right) \mathrm{d} a^{\prime}
$$

a condition that guarantees the existence of age-structured two-sex mixing functions (Castillo-Chavez, Huang \& Li 1994). The only separable two-sex mixing function (Ross solution) satisfying properties (B1)-(B3), or the so-called Ross solution, is given by

$$
\bar{p}\left(a^{\prime}, t, \cdot\right)=\frac{B\left(a^{\prime}, \cdot\right) F\left(a^{\prime}, t\right)}{\int_{0}^{\infty} B\left(u^{\prime}, \cdot\right) F\left(u^{\prime}, t\right) \mathrm{d} u^{\prime}} \quad \text { and } \quad \bar{q}(a, t, \cdot)=\frac{C(a, \cdot) M(a, t)}{\int_{0}^{\infty} C(u, \cdot) M(u, t) \mathrm{d} u}
$$


This separable function models a heterosexually age-structured randomly (proportionate) mixing twosex population.

Castillo-Chavez and Busenberg (1991) prove that all two-sex mixing functions for a population with distinct subgroups can be characterized as multiplicative perturbations of the corresponding separable solution. Similarly for an age-structured population all solutions to axioms (B1)-(B3) can also be characterized as multiplicative perturbations of the separable solution (23). To describe this formulation, we let $\phi^{m}\left(a, a^{\prime}\right)$ denote the preference or affinity of males of age $a$ for females of age $a^{\prime}$, and $\phi^{f}\left(a^{\prime}, a\right)$ denote the preference or affinity of females of age $a^{\prime}$ for males of age $a$. The affinities can be dependent of time, or change with time and/or group sizes. (We omit this level of detail below to simplify the notation.) The following definitions are needed.

$$
\begin{aligned}
R^{m}(a) & \equiv 1-\int_{0}^{\infty} \bar{p}\left(a^{\prime}\right) \phi^{m}\left(a, a^{\prime}\right) \mathrm{d} a^{\prime}=\text { average disaffinity of males with age } a \\
R^{f}\left(a^{\prime}\right) & \equiv 1-\int_{0}^{\infty} \bar{q}(a) \phi^{f}\left(a^{\prime}, a\right) \mathrm{d} a=\text { average disaffinity of females with age } a^{\prime}, \\
V^{m} & \equiv \int_{0}^{\infty} \bar{q}(a) R^{m}(a) \mathrm{d} a=\text { weighted average disaffinity of males, } \\
V^{f} & \equiv \int_{0}^{\infty} \bar{p}\left(a^{\prime}\right) R^{f}\left(a^{\prime}\right) \mathrm{d} a^{\prime}=\text { weighted average disaffinity of females. }
\end{aligned}
$$

In principle the functions $\phi^{m}$ and $\phi^{f}$ can take on any values, positive or negative, which make $R^{m} \geq 0$, $R^{f} \geq 0, V^{m}>0$ and $V^{f}>0$ at all times. If one adds the additional restriction that $0 \leq R^{m} \leq 1$ and $0 \leq R^{f} \leq 1$, then these functions can take on only non-negative values (Hsu Schmitz 1994). Now we can express the representation theorem of two-sex mixing function in an age-structured population:

\section{Theorem 5 .}

For each two-sex mixing function $(p, q)$ at any time, non-negative affinity functions $\phi^{m}\left(a, a^{\prime}\right)$ and $\phi^{f}\left(a^{\prime}, a\right)$ can be found so that for $a \in[0, \infty)$ and $a^{\prime} \in[0, \infty)$

$$
\begin{aligned}
& p\left(a, a^{\prime}\right)=\bar{p}\left(a^{\prime}\right)\left[\frac{R^{f}\left(a^{\prime}\right) R^{m}(a)}{V^{f}}+\phi^{m}\left(a, a^{\prime}\right)\right], \\
& q\left(a^{\prime}, a\right)=\bar{q}(a)\left[\frac{R^{m}(a) R^{f}\left(a^{\prime}\right)}{V^{m}}+\phi^{f}\left(a^{\prime}, a\right)\right],
\end{aligned}
$$


if and only if

$$
\phi^{m}\left(a, a^{\prime}\right)=\phi^{f}\left(a^{\prime}, a\right)+R^{m}(a) R^{f}\left(a^{\prime}\right)\left[\frac{1}{V^{m}}-\frac{1}{V^{f}}\right]
$$

where $0 \leq R^{m}(a) \leq 1$ for all $a, \quad 0 \leq R^{f}\left(a^{\prime}\right) \leq 1$ for all $a^{\prime}$, but not all $R^{m}(a)$ and not all $R^{f}\left(a^{\prime}\right)$ can be zeros.

The incorporation of affinity/preference structure is not intended for the purpose of contructing complex mixing patterns but rather to make them richer and closer to reality. In fact, this view and the representation theorem brings population biology models closer to those in the existing literature on mating functions. (Population geneticists, e.g., Wilson (1973), Wagener (1976), Karlin (1979a, b, c, d, 1980), Burley (1983) and Gimelfarb (1988a, b), have long recognized the important role of preferences in mating patterns.)

Condition in (25) exhibits a relationship between male affinity $\phi^{m}$ and female affinity $\phi^{f}$. It might be possible to obtain $\phi^{m}$ in terms of $\phi^{f}, \bar{p}$ and $\bar{q}$, that is, to find the function $\Psi$ such that $\phi^{m}=\Psi\left(\phi^{f}, \bar{p}, \bar{q}\right)$. Since we are dealing with a continuous variable, age, directly solving the non-linear equation system for $\phi^{m}$ is not feasible (infinite dimensional system). Nevertheless, we can still establish the same results as in the discrete case.

First a frequency-independent function $\Psi$ can be easily derived as expressed in the following theorem:

\section{Theorem 6.}

$$
V^{f}=V^{m} \text { if and only if } \phi^{m}\left(a, a^{\prime}\right)=\phi^{f}\left(a^{\prime}, a\right) \text { for } a \in[0, \infty) \text { and } a^{\prime} \in[0, \infty) \text {. }
$$

The proof is simple and given in Hsu Schmitz (1994), and Hsu Schmitz et al. (1994). This theorem defines a special $\Psi$ function:

$$
\phi^{m}\left(a, a^{\prime}\right)=\Psi\left[\phi^{f}\left(a^{\prime}, a\right), \bar{p}, \bar{q}\right]=\phi^{f}\left(a^{\prime}, a\right),
$$

which is independent of $\bar{p}$ and $\bar{q}$, i.e., frequency independent. Although the class of solutions with $\phi^{m}\left(a, a^{\prime}\right)=\phi^{f}\left(a^{\prime}, a\right)$ is quite restrictive, this class extends considerably the age-dependent mixing/mating structures available in the literature. Furthermore, the class of parametric mixing models becomes quite rich and flexible as the use of preference function $\phi\left(a, a^{\prime}\right)=v \exp \left[-\kappa\left(a-a^{\prime}\right)^{2}\right]$ clearly shows. 
To derive the general $\Psi$ function results on integral equations are required (the Fredholm alternative). The details are provided in Hsu Schmitz (1994) and Hsu Schmitz et al. (1994). The final results are collected in the following theorem:

\section{Theorem 7. (age-structured $\mathrm{T}^{3}$ Theorem)}

The non-negative affinities of two genders, $\phi^{m}\left(a, a^{\prime}\right)$ and $\phi^{f}\left(a^{\prime}, a\right)$ for $a \in[0, \infty)$ and $a^{\prime} \in[0, \infty)$, obey the following explicit relation at all times:

$$
\phi^{m}\left(a, a^{\prime}\right)=\phi^{f}\left(a^{\prime}, a\right)+\left(1-\frac{\lambda}{V^{f}}\right) \frac{\left[1-U^{f}(a)\right] R^{f}\left(a^{\prime}\right)}{V^{f}},
$$

where $U^{f}(a) \equiv \int_{0}^{\infty} \bar{p}\left(a^{\prime}\right) \phi^{f}\left(a^{\prime}, a\right) \mathrm{d} a^{\prime} \leq 1$ for $a \in[0, \infty)$ but not all $U^{f}(a)$ can be 1 , and $\lambda$ is an arbitrary constant within the frequency-dependent range

$$
0<\lambda \leq \frac{V^{f}}{1-\min _{a} U^{f}(a)} .
$$

Conversely,

$$
\phi^{f}\left(a^{\prime}, a\right)=\phi^{m}\left(a, a^{\prime}\right)+\left(1-\frac{\gamma}{V^{m}}\right) \frac{\left[1-U^{m}\left(a^{\prime}\right)\right] R^{m}(a)}{V^{m}},
$$

where $U^{m}\left(a^{\prime}\right) \equiv \int_{0}^{\infty} \bar{q}(a) \phi^{m}\left(a, a^{\prime}\right) \mathrm{d} a \leq 1$ for $a^{\prime} \in[0, \infty)$ but not all $U^{m}\left(a^{\prime}\right)$ can be 1 , and $\gamma$ is an arbitrary constant within the frequency-dependent range

$$
0<\gamma \leq \frac{V^{m}}{1-\min _{a^{\prime}} U^{m}\left(a^{\prime}\right)}
$$

Expression (27) explicitly defines the general $\Psi$ function, while expression (29) defines the general function needed to obtain $\phi^{f}$ for given $\phi^{m}, \bar{p}$ and $\bar{q}$. However, for a given $\phi^{f}$ or $\phi^{m}$ several corresponding $\phi^{m}$ or $\phi^{f}$ are possible. For example, if $\lambda=V^{f}$ or $\gamma=V^{m}$, then $\phi^{m}\left(a, a^{\prime}\right)=\phi^{f}\left(a^{\prime}, a\right)$ for all $a$ and $a^{\prime}$, and Theorem 6 is recovered.

If $\phi^{f}\left(a^{\prime}, a\right)=\alpha$, where $\alpha$ is a constant and $0 \leq \alpha<1$, for all $a$ and $a^{\prime}$, i.e., females have no specific preferences for males of different ages, then $0<R^{f}\left(a^{\prime}\right)=1-\alpha \leq 1$ for all $a^{\prime}, 0 \leq U^{f}(a)=$ $\alpha<1$ for all $a$, and $0<V^{f}=1-\alpha \leq 1$. Hence, equation (27) becomes

$$
\phi^{m}\left(a, a^{\prime}\right)=\alpha+\left(1-\frac{\lambda}{1-\alpha}\right)(1-\alpha)=1-\lambda
$$

for all $a$ and $a^{\prime}$, where $1-\lambda$ is a constant and $0 \leq 1-\lambda<1$ (males have no specific preferences for females of different ages, either). Furthermore, $0<R^{m}(a)=\lambda \leq 1$ for all $a$ and $0<V^{m}=\lambda \leq 1$. Thus, 
the mixing function in (24) reduces to the Ross solution, the heterosexually random mixing function.

With $\phi^{m}$ replaced by (27), the two-sex mixing function in (24) becomes

$$
\begin{aligned}
& p\left(a, a^{\prime}\right)=\bar{p}\left(a^{\prime}\right)\left\{\frac{\left[1-U^{f}(a)\right] R^{f}\left(a^{\prime}\right)}{V^{f}}+\phi^{f}\left(a^{\prime}, a\right)\right\}, \\
& q\left(a^{\prime}, a\right)=\bar{q}(a)\left\{\frac{\left[1-U^{f}(a)\right] R^{f}\left(a^{\prime}\right)}{V^{f}}+\phi^{f}\left(a^{\prime}, a\right)\right\} .
\end{aligned}
$$

The free parameters $\lambda$ or $\gamma$ are absent in (31). This implies that for a given female affinity $\phi^{f}$, the corresponding male affinity $\phi^{m}$ can take several behavioral "genotypes" represented by distinct $\lambda$, which all result in the same behavioral "phenotype" $(p, q)$ as expressed in (31). Equivalent results can be obtained using the male affinity $\phi^{m}$ as given. Again, this finding agrees with Burley's (1983) statement that different preferences can result in identical mating patterns.

\section{Conclusions}

In this chapter we have introduced a modeling framework that allows for the modeling of mixing/mating density dependent conditional probabilities in heterosexual populations. The incorporations of the probabilites in either stochastic or deterministic demographic or epidemic models is straightforward (see Castillo-Chavez, Fridman and Luo 1995, Castillo-Chavez, Huang and Li 1995).

Several results have been established that have generated useful insights in the fields of population dynamics and epidemiology of populations with mating systems. For example, for pair formation models with an arbitrary number of types we (Castillo-Chavez, Huang and Li 1995) have established that the distribution of the average times spent in partnerships/pairs plays a central role in determining the observed data on the the distributions of pairs. If there is very little variation, that is, if most types of individuals remain in pairings approximately the same amount of time then the observed data on the distribution of pairs may reflect the actual mating system with high probability. If, on the other hand, the resident time in partnerships is highly variable then the observed data on the distributions of pair types (who pairs with whom) will not reflect the mating system with high probability at all. 
Further theoretical studies are needed to better understand the role of mating systems in population biology. We hope that this chapter will motivate further theoretical work in this area.

\section{References}

M. Altman and M. Morris (1994). A clarification of the $\phi$ mixing model. Math. Biosci. 124 (1): 1-8. Blythe, S. P., Castillo-Chavez, C., Palmer, J., Cheng, M.: Towards a unified theory of mixing and pair formation. Math. Biosci. 107, 379-405 (1991)

Blythe, S. P., Busenberg, S., Castillo-Chavez, C.: Affinity in paired event probability (1995). Math. Biosci. 128: 265-284.

Burley, N.: The meaning of assortative mating. Ethology and Sociobiology 4, 191-203 (1983)

Busenberg, S., Castillo-Chavez, C.: Interaction, pair formation and force of infection terms in sexuallytransmitted diseases. In: C. Castillo-Chavez: Mathematical and Statistical Approaches to AIDS Epidemiology (Lect. Notes Biomath. vol. 83, pp.289-300) Berlin, Heidelberg, New York, London, Paris, Tokyo, Hong Kong: Springer 1989

Busenberg, S., Castillo-Chavez, C.: A general solution of the problem of mixing sub-populations, and its application to risk- and age-structured epidemic models for the spread of AIDS. IMA J. Math. Appl. Med. \& Biol. 8, 1-29 (1991)

Castillo-Chavez, C. (Ed.): Mathematical and Statistical Approaches to AIDS Epidemiology (Lect. Notes Biomath. vol. 83) Berlin, Heidelberg, New York, London, Paris, Tokyo, Hong Kong: Springer 1989.

Castillo-Chavez, C., Busenberg, S.: On the solution of the two-sex mixing problem. In: S. Busenberg, M. Martelli: Proceedings of the International Conference on Differential Equations and Applications to Biology and Population Dynamics (Lect. Notes Biomath. vol. 92, pp.80-98) Berlin, Heidelberg, New York, London, Paris, Tokyo, Hong Kong, Barcelona, Budapest: Springer 1991.

Castillo-Chavez, C., Busenberg, S., Gerow, K.: Pair formation in structured populations. In: J. Goldstein, F. Kappel, W. Schappacher: Differential Equations with Applications in Biology, Physics and Engineering (pp.47-65) New York: Marcel Dekker 1991. 
Castillo-Chavez, C., Fridman, S., Luo, X.: Stochastic and deterministic models in epidemiology. In: Proceedings of the First World Congress of Non-Linear Analysts (Tampa, Florida, August 19-26, 1992) Berlin: Walter de Gruyter \& Co. (In press.)

Castillo-Chavez, C., Huang, W., Li, J.: On the existence of stable pairing distributions (1995). J. Math. Biol. (in press).

Castillo-Chavez, C., Shyu, S.-F., Rubin, G., Umbach, D.: On the estimation problem of mixing/pair formation matrices with applications to models for sexually-transmitted diseases. In: K. Dietz et al.: AIDS Epidemiology: Methodology Issues (pp.384-402) Boston, Basel, Berlin: Birkhäuser 1992.

Castillo-Chavez, C., Velasco-Hernandez, J. X., Fridman, S.: Modeling contact structures in biology (Lect. Notes Biomath. vol. 100): Springer (1995).

Caswell, H. and D.E. Weeks (1986). Two-sex models: chaos, extinction and other dynamic consequences of sex. American Naturalist 128: 707-735.

Crow, J. F., Kimura, M.: An Introduction to Population Genetics Theory. New York: Harper and Row 1970.

Fredrickson, A. G.: A mathematical theory of age structure in sexual populations: Random mating and monogamous marriage models. Math. Biosci. 10, 117-143 (1971).

Gimelfarb, A.: Processes of pair formation leading to assortative mating in biological populations: Encounter-mating model. The American Naturalist 131, 865-884 (1988a).

Gimelfarb, A.: Processes of pair formation leading to assortative mating in biological populations: Dynamic interaction model. Theoretical Population Biology 34, 1-23 (1988b).

Hsu Schmitz, S.-F.: Some theories, estimation methods and applications of marriage functions in demography and epidemiology (Ph. D. dissertation) Ithaca, New York: Cornell University 1994.

Hsu Schmitz, S.-F., Busenberg, S., Castillo-Chavez, C.: On the evolution of marriage functions: it takes two to tango. Biometrics Unit Technical Report, BU-1210-M, Cornell University, Ithaca, New York (1993).

Hsu Schmitz, S.-F., Castillo-Chavez, C.: Completion of mixing matrices for non-closed social networks. In: Proceedings of the First World Congress of non-linear Analysts (Tampa, Florida, August 19-26, 1992) Berlin: Walter de Gruyter \& Co. (In press.) 
Hsu Schmitz, S.-F., Castillo-Chavez, C.: Parameter estimation in non-closed social networks related to dynamics of sexually transmitted diseases. In: E. Kaplan, M. Brandeau: Modeling the AIDS Epidemic: Planning, Policy and Prediction (pp.533-559) New York: Raven Press 1994.

Karlin S.: Models of multifactorial inheritance: I, Multivariate formulations and basic convergence results. Theoretical Population Biology 15, 308-355 (1979a).

Karlin S.: Models of multifactorial inheritance: II, The covariance structure for a scalar phenotype under selective assortative mating and sex-dependent symmetric parental-transmission. Theoretical Population Biology 15, 356-393 (1979b).

Karlin S.: Models of multifactorial inheritance: III, Calculation of covariance of relatives under selective assortative mating. Theoretical Population Biology 15, 394-423 (1979c).

Karlin S.: Models of multifactorial inheritance: IV, Asymmetric transmission for a scalar phenotype. Theoretical Population Biology 15, 424-438 (1979d).

Karlin S.: Models of multifactorial inheritance: V, Linear assortative mating as against selective (nonlinear) assortative mating. Theoretical Population Biology 17, 255-275 (1980).

Kendall, D. G.: Stochastic processes and population growth. J. Royal Stat. Soc. B 11, 230-264 (1949). Keyfitz, N.: The mathematics of sex and marriage. Proceedings of the Sixth Berkeley Symposium on Mathematical Statistics and Probability 4, 89-108 (1949).

Lubkin, S., Castillo-Chavez, C.: A pair formation approach to modeling inheritance of social traits. In: Proceedings of the First World Congress of Non-Linear Analysts (Tampa, Florida, August 19-26, 1992) Berlin: Walter de Gruyter \& Co. (In press.)

McFarland, D. D.: Comparison of alternative marriage models. In: T. N. E. Greville: Population Dynamics (pp.89-106) New York, London: Academic Press 1972.

Parlett, B.: Can there be a marriage function. In: T. N. E. Greville: Population Dynamics (pp.107-135) New York, London: Academic Press 1972.

Pollard, J. H.: Mathematical Models for the Growth of Human Populations. Cambridge: Cambridge University Press 1973. 
Rubin, G., Umbach, D., Shyu, S.-F., Castillo-Chavez, C.: Application of capture-recapture methodology to estimation of size of population at risk of AIDS and/or other sexually-transmitted diseases. Stat. in Med. 11, 1533-1549 (1992).

Wagener D. K.: Preferential mating: Nonrandom mating of a continuous phenotype. Theoretical Population Biology 10, 185-204 (1976).

Wilson, S. R.: The correlation between relatives under the multifactorial models with assortative mating: I, The multifactorial model with assortative mating. Annals of Human Genetics 37, 289-304 (1973). 
Figure 1. Mixing matrix estimated from data.
(a) Male, $\hat{\mathbf{P}}$; (b) Female, $\hat{\mathbf{Q}}$.

Figure 2. Random mixing matrix for our data.

(a) Male, $\overline{\mathbf{P}}$; (b) Female, $\overline{\mathbf{Q}}$. 


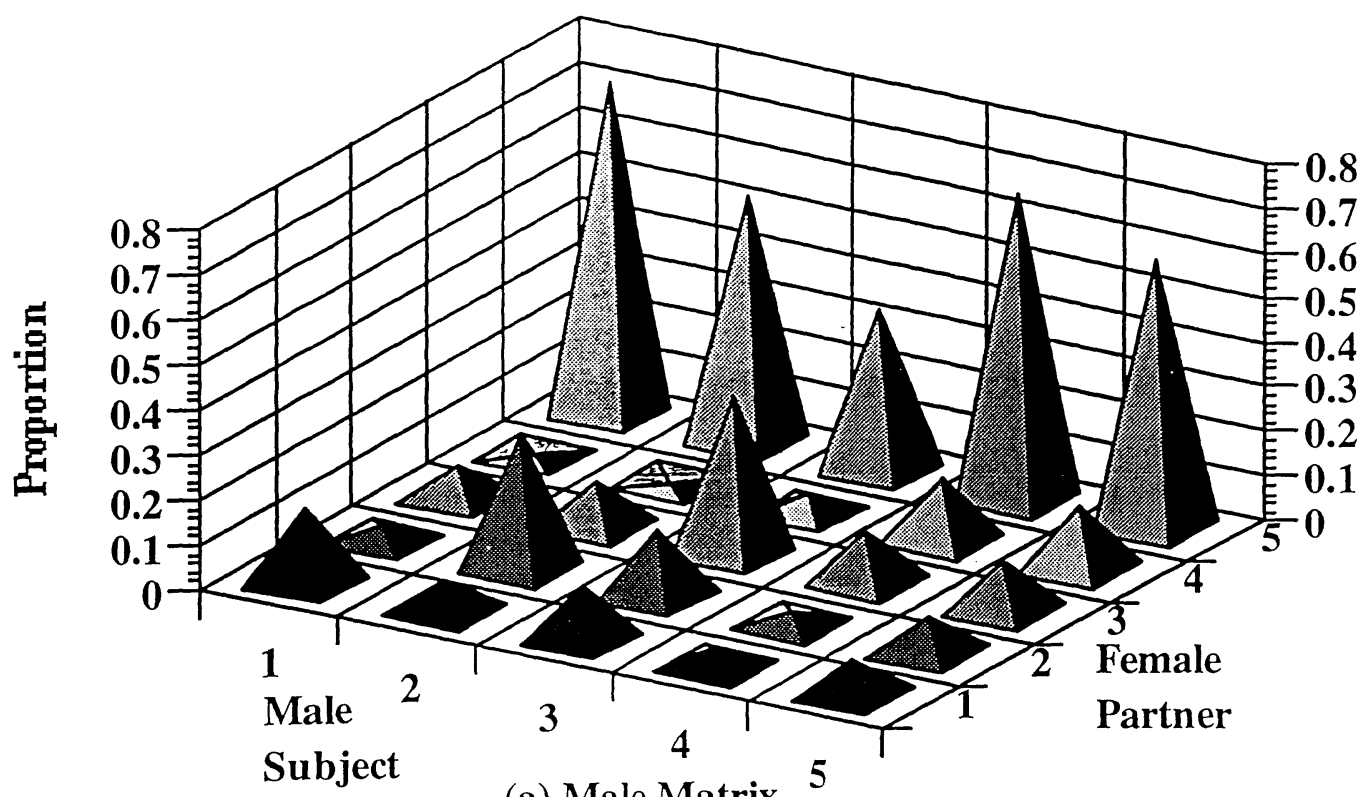

(a) Male Matrix 5

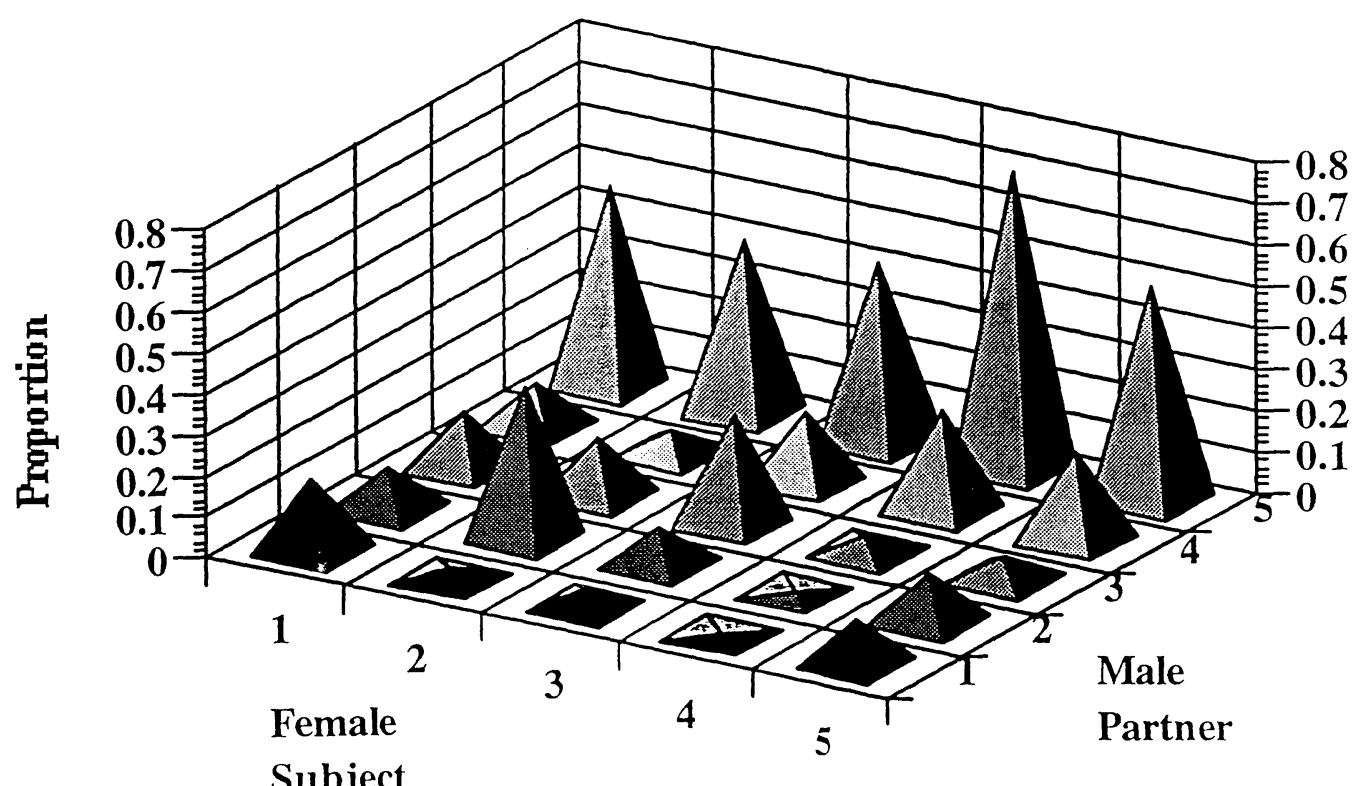

(b) Female Matrix

Figure 1. 


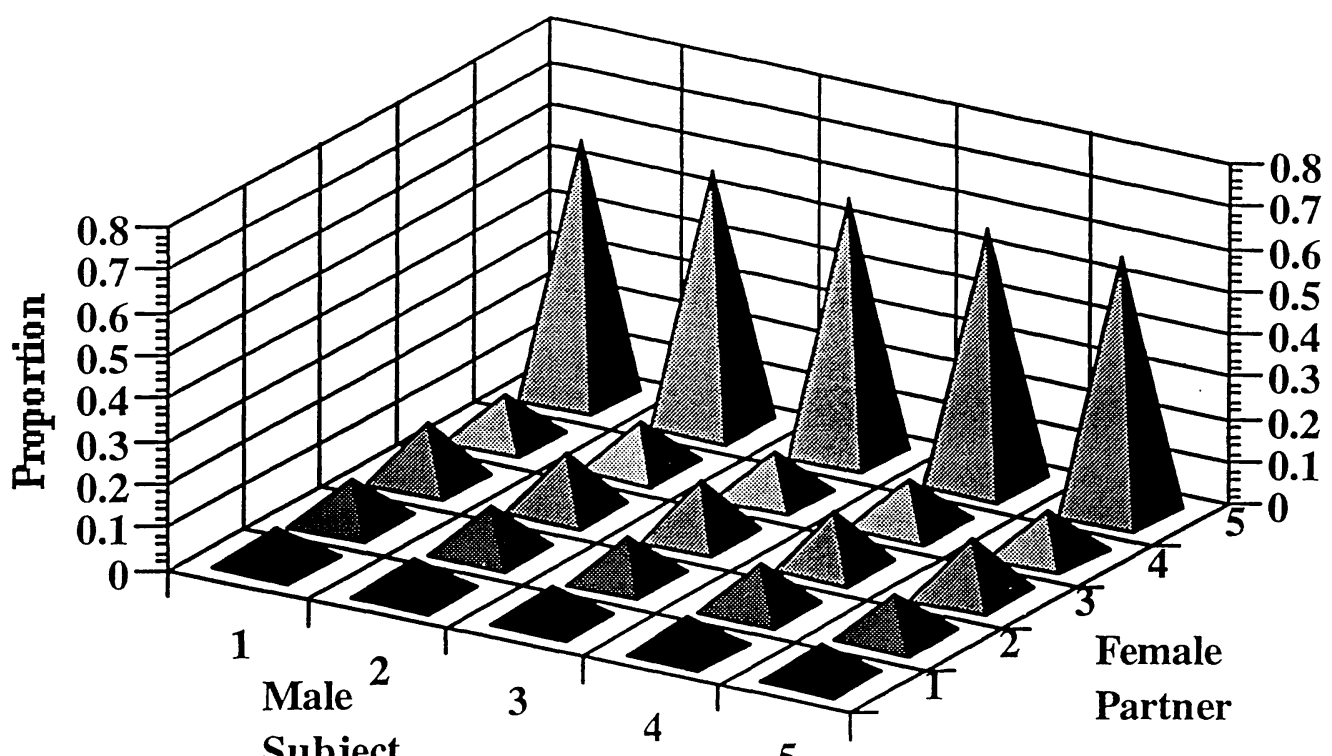

(a) Male Matrix 5

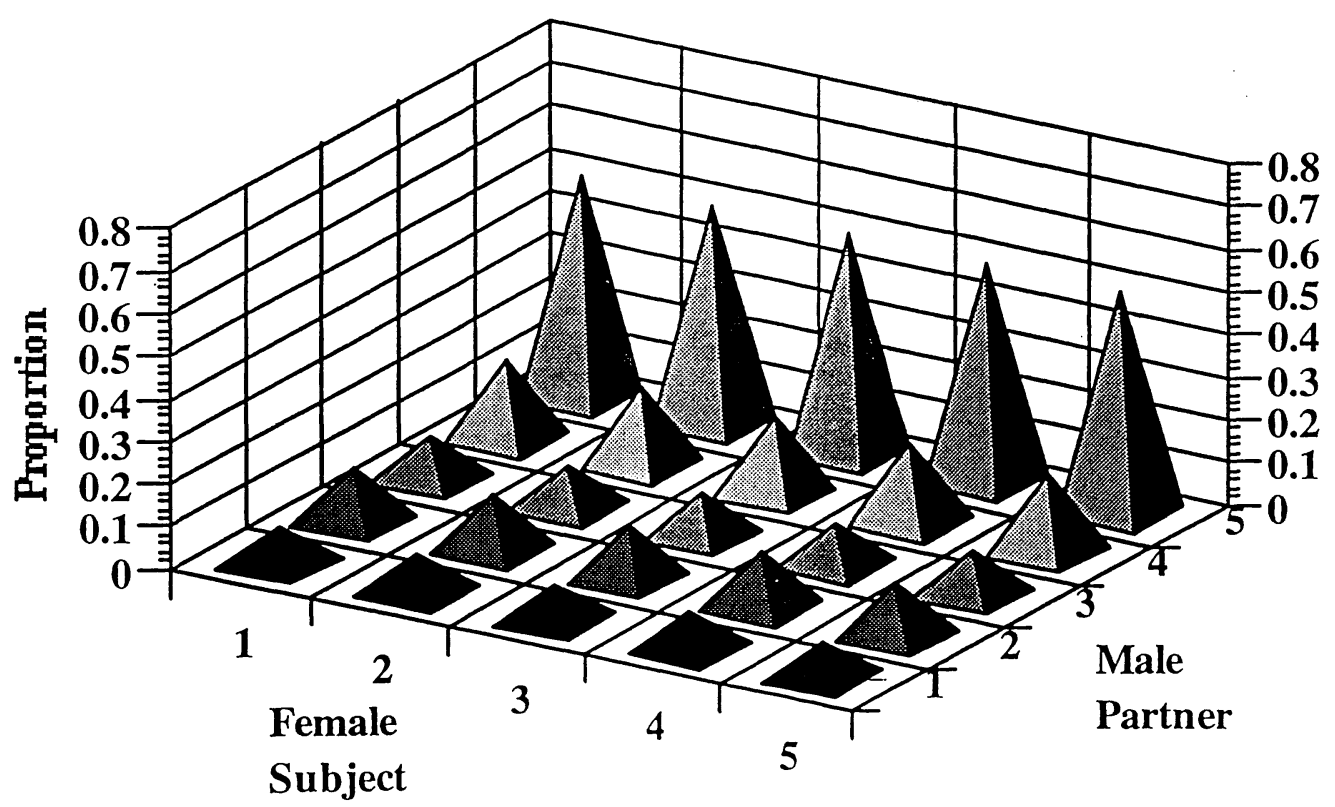

(b) Female Matrix

Figure 2. 